\title{
Anorexia nerviosa: La eficacia de la terapia de conducta*
}

\section{J. Kellerman}

\section{RESUMEN:}

Dos articulos criticos sobre el tratamiento conductual de la anorexia nerviosa publicados por la Dra. Bruch (1976), en los que afirma que el peso ganado por este procedimiento se pierde rápidamente y que esta ganancia de peso no se acompaña de otros cambios positivos, se compara con la revisión de varios artículos en los que se utilizó la terapia de conducta en este trastorno. Las críticas de Bruch no son apoyadas por los datos. Se observa la existencia de pruebas empíricas que apoyan la idea de que la terapia de conducta es el tratamiento de elección en la anorexia nerviosa.

En un artículo reciente, Bruch (1976) criticaba el uso de técnicas de modificación de conducta en la anorexia nerviosa. Estas críticas eran similares a las presentadas en un artículo anterior (Bruch, 1974). A pesar de los grandes conocimientos de la autora en el área de los trastornos de comida y sus numerosas publicaciones (Bruch 1962, 1963, 1966, 1971), su crítica es muy pobre, especialmente en lo que se refiere a datos controlados. Este estudio intenta probar los supuestos planteados por la Dra. Bruch (1976) comparando sus críticas con los tratamientos conductuales de anorexia que se han publicado.
Características de las críticas de Bruch (1976)

Las criticas de Bruch pueden dividirse en dos apartados:

1. Los éxitos obtenidos con la «modificación de conducta» para conseguir una ganancia de peso son engañosos, debido a no existir seguimientos suficientemente largos.

2. La ganancia de peso "per se» no es un criterio digno de confianza respecto al "progreso» terapéutico. A menos que los problemas subyacentes se mejoren, el paciente sufrirá y a veces morirá.

\section{ESTUDIO DE CASOS}

Se han utilizado todos los estudios que utilizaron modificación de conducta en anorexia nerviosa, publicados durante los 10 últimos años en Bebavior Reserch and Therapy, y durante los últimos 7 años en The Journal of Behavior Therapy and Experimental Psychiatry. Estos estudios se examinaron ateniéndose a los siguientes criterios:

a) existencia de un seguimiento «largo y especificado en el artículo».

b) información de cambios psicológicos concomitantes. Específicamente

* "Anorexia nervosa: efficacy of bebavior therapy", Journal of Babavioral Therapy and Experimental Psychiatry, 1977, vol. 8, pp. $387-390$. Reproducido con autorización. (S) De esta traducción, Estudios de Psicologia. 
los que se referian a cambios en el ajuste psicológico. Se anotaron también las muertes, debido a que la $\mathrm{Dr}$ Bruch $(1974,1976)$ señalaba este resultado en relación con el uso de la terapia de conducta en anorexia nerviosa.

\section{RESULTADOS}

Los resultados se detallan en la tabla 1.

En general los datos no apoyan los asertos de la Dra. Bruch respecto a la falta de seguimiento y a la rápida caída postratamiento, de los 11 estudios revisados 9 (13 pacientes) presentaban datos de seguimiento que oscilaban entre los cuatro y los 30 meses, con una media de seguimiento de doce con nueve meses. Nueve de los 13 pacientes mantenian una ganancia de peso total, 3 mantenían una ganancia de peso parcial y una habia muerto por suicidio a las semanas de iniciarse el tratamiento.

En 9 pacientes se observaron cambios positivos concomitantes, incluyendo mejoría en el ajuste social e incremento de autoestima. En un paciente se señaló una interacción social con pocas defensas, pero no hubo evidencia de si se debia a su etapa de desarrollo o era resultado del tratamiento.

Así pues, no se confirmó el segundo aserto de la Dra. Bruch, en que criticaba el tratamiento conductual de la anorexia

TABLA 1.

Seguimiento y cambios concomitantes como resultado del tratamiento conductual en la anorexia nerviosa.

\begin{tabular}{|c|c|c|c|c|c|}
\hline Estudio & $\begin{array}{l}\mathrm{N} .^{\circ} \text { de } \\
\text { pacientes }\end{array}$ & $\begin{array}{l}\text { Periodo } \\
\text { seguimiento }\end{array}$ & Resultados & $\begin{array}{l}\text { Otros } \\
\text { cambios }\end{array}$ & $\begin{array}{l}\text { Terapia } \\
\text { complementaria }\end{array}$ \\
\hline $\begin{array}{l}\text { Bachrach } \\
\text { (1965) }\end{array}$ & 1 & 18 meses & $\begin{array}{l}\text { ganancia } \\
\text { peso-parcial }\end{array}$ & $\begin{array}{l}\text { Incremento } \\
\text { interacción } \\
\text { social }\end{array}$ & $\begin{array}{l}\text { Sesiones } \\
\text { terapéuticas } \\
\text { ambientales }\end{array}$ \\
\hline $\begin{array}{l}\text { Hallsten } \\
(1965)\end{array}$ & 1 & 5 meses & $\begin{array}{l}\text { ganancia } \\
\text { peso parcial }\end{array}$ & $\begin{array}{l}\text { Buen } \\
\text { ajuste }\end{array}$ & $\begin{array}{l}\text { Terapia } \\
\text { familiar }\end{array}$ \\
\hline $\begin{array}{l}\text { Lang } \\
\text { (1965) }\end{array}$ & 1 & 12 meses & $\begin{array}{l}\text { ganancia } \\
\text { peso parcial }\end{array}$ & $\begin{array}{l}\text { Incremento } \\
\text { Ajuste social }\end{array}$ & Ninguna \\
\hline Leintemberg & 2 & 9 meses & ganancia & A juste laboral & Ninguna \\
\hline (1968) & & 4 meses & peso parcial & Ajuste laboral & Ninguna \\
\hline $\begin{array}{l}\text { Azzerad y } \\
\text { Stafford } \\
(1969)\end{array}$ & 1 & 5 meses & $\begin{array}{l}\text { ganancia } \\
\text { peso parcial }\end{array}$ & Ninguno & Ninguna \\
\hline $\begin{array}{l}\text { Stumphanzer } \\
\text { (1969) }\end{array}$ & 1 & 10 dias & $\begin{array}{l}\text { ganancia } \\
\text { peso continuada }\end{array}$ & $\begin{array}{l}\text { Incremento } \\
\text { cooperación }\end{array}$ & Ninguna \\
\hline \multirow[t]{3}{*}{$\begin{array}{l}\text { Blinder } \\
(1970)\end{array}$} & 3 & $\begin{array}{l}\text { a) varias } \\
\text { semanas }\end{array}$ & $\begin{array}{l}\text { ganancia } \\
\text { peso parcial }\end{array}$ & Suicidio & $\begin{array}{l}\text { Terapia } \\
\text { apoyo }\end{array}$ \\
\hline & & b) 10 meses & $\begin{array}{l}\text { ganancia } \\
\text { peso total }\end{array}$ & $\begin{array}{l}\text { Ajuste } \\
\text { escolar }\end{array}$ & Ninguna \\
\hline & & c) 8 meses & $\begin{array}{l}\text { ganancia } \\
\text { peso parcial }\end{array}$ & $\begin{array}{l}\text { Defensiva en } \\
\text { relaciones } \\
\text { sociales }\end{array}$ & Psicoterapia \\
\hline \multirow[t]{2}{*}{$\begin{array}{l}\text { Bianco } \\
(1970)\end{array}$} & 2 & & $\begin{array}{l}\text { ganancia } \\
\text { peso total }\end{array}$ & Buen ajuste & $\begin{array}{l}\text { Terapia } \\
\text { apoyo }\end{array}$ \\
\hline & & b) 24 meses & $\begin{array}{l}\text { ganancia } \\
\text { peso total }\end{array}$ & Buen ajuste & $\begin{array}{l}\text { Terapia } \\
\text { apoyo }\end{array}$ \\
\hline \multirow{3}{*}{$\begin{array}{l}\text { Schunrer } \\
\text { (1973) } \\
\text { Agras } \\
\text { (1974) } \\
\text { Neumann y } \\
\text { Gaoni } \\
(1975)\end{array}$} & 1 & 6 meses & $\begin{array}{l}\text { ganancia } \\
\text { peso total }\end{array}$ & Buen ajuste & $\begin{array}{l}\text { Terapia } \\
\text { apoyo }\end{array}$ \\
\hline & 7 & Ninguno & $\begin{array}{l}\text { ganancia } \\
\text { peso parcial }\end{array}$ & Ninguna & Ninguna \\
\hline & 1 & 30 meses & $\begin{array}{l}\text { ganancia } \\
\text { peso total }\end{array}$ & Buen ajuste & Ninguna \\
\hline
\end{tabular}


nerviosa, y en donde afirmaba que la ganancia de peso, como meta de tratamiento exclusiva, no estaba relacionada con cambios positivos en otras áreas, y que incluso llevaba a la muerte. En los artículos revisados ninguno de los pacientes tratados murió como resultado de su trastorno y en la mayoría se informó de signos de mejoría en el ajuste psicológico. El suicidio parece que estuvo en relación con una conversación entre la paciente y su madre. No existe evidencia de que el tratamiento provocara el suicidio, a pesar de lo dicho por la Dr Bruch (1974).

\section{DISCUSION}

¿Por qué existe esa discrepancia entre las afirmaciones de la Dra. Bruch y los datos de estos estudios? Sólo se puede especular sobre la influencia de las creencias teóricas en las actitudes de los clínicos, incluso tan sofisticados y conocedores del tema como la Dra. Bruch. Ella (Bruch, 1974, 1976) presenta casos en que la modificación de conducta no fue útil para establecer $\mathrm{y}$ mantener el peso de pacientes anoréticas. Conviene examinar estos casos para delimitar las variables fundamentales de un tratamiento eficaz. Primero, un examen de las criticas de Bruch (1974, 1976), indican que su uso del término «modificación de conducta" no es correcto. La descripción detallada que ofrece la Dra. Bruch indica una falta de familiaridad y conocimiento de los procedimientos de terapia de conducta. La paciente que «situada en otro programa de modificación de conducta, donde la comida era reforzada con la amenaza de intubación (tres tubos de comida) cada día que fallara en alcanzar la ganancia de peso prescritas. Este programa de tratamiento implica refuerzo negativo, es decir, un intento de incrementar la conducta mediante la evitación de las consecuencias aversivas. Esto difiere de los estudios revisados sobre modificación de conducta que utilizaban refuerzos positivos, procedimientos de coste de respuesta y reducción de la ansiedad relacionada con la comida. La descripción de Bruch de que la comida se reforzaba por una amenaza es errónea. En un sistema operante, la conducta se supone que se controla por sus consecuencias, no por la amenaza de estas consecuencias.

Es curioso señalar que, en el mismo trabajo, la Dra. Bruch (1976) utiliza la amenaza para una de sus pacientes: «Se dijo también que Mary sería hospitalizada si no se apreciaba una ganancia de peso en poco tiempo.» Esta manifestación de contingencia produjo una ganancia de peso y «la situación parecía mejorada globalmente». El parentesco entre los métodos de Bruch y los tratamientos conductuales ha sido ya señalado por Wolpe (1975).

A pesar de las críticas a la terapia de conducta, considerada como inútil para el tratamiento de la anorexia nerviosa, los terapeutas de conducta recibirían gustosos cualquier sugerencia útil. Cuando examinamos lo que Bruch ofrece, nos encontramos con los siguiente: «El éxito del tratamiento exige la integración de varias tareas distintas: 1) solución de los problemas psicológicos subyacentes. 2) corrección de las relaciones familiares patológicas. 3) restablecimiento de la nutrición normal.»

Mientras que todo terapeuta sensato estaria de acuerdo con estas metas, le gustaría un poco más de información para saber cómo conseguirlas.

Varios de los estudios revisados incluían terapias complementarias además de los procedimientos conductuales, las cuales se realizaron una vez que se había conseguido ganar peso.

Sin embargo, 6 de los estudios que habían tenido éxito, no informaron del uso de psicoterapia complementaria.

Otro aspecto de la critica de Bruch, señalado por Wolpe (1975), es la diferencia entre condenar una teoría que fracasa y condenar los fracasos cuando la teoría se utiliza de forma inadecuada. La terapia de conducta implica un análisis conductual que logre información sobre los 
estímulos antecedentes de la conducta. Esto incluye el estudio de las respuestas de ansiedad; como Wolpe (1975) señala, un tratamiento que no considera ni modifica las respuestas de ansiedad existentes, debe considerarse como fracaso, aunque logre modificar las conductas secundarias a ella. Este pudo ser el error fatal en alguno de los casos descritos por Bruch $(1974,1976)$. La mayoría de las publicaciones recogidas en este estudio utilizaban un sistema operante para incrementar la ingesta de comida o el peso. El éxito pudo haber estado en relación con la no existencia de ansiedad en estos pacientes. Sin embargo, dos estudios (Hallsten 1965, Schnurer y col. 1973) trataron la anorexia nerviosa como una respuesta de ansiedad relacionada con la comida. Así su tratamiento fue la desensibilización sistemática. Lo fundamental es señalar que la forma de tratamiento viene dada por el análisis conductual. Los métodos que logran liberar al individuo de sus conductas aprendidas desadaptadas, llevan, en contra de lo que opina Bruch, a una mayor autonomía y satisfacción posterior. Blinder y col. (1970) han comparado el tratamiento conductual de la anorexia nerviosa con otros métodos y han encontrado que dicho método es el que tiene más éxito en conseguir el aumento de peso de las pacientes. Nuestro estudio presenta datos que demuestran que esta ganancia de peso se mantiene a largo plazo.

Hay datos, y no argumentos emocionales, para afirmar que la terapia de conducta parece ser el tratamiento de elección para la anorexia nerviosa.

\section{Referencias}

Agras W. S. et al.: «Behavior modification of anorexia nervosa». Arcb. Gen. Psycbiat. 30, 279-286.

AZERRAD J., and STAFFORD, R. L.: «Restoration of eating behavior in anorexia nervosa through operant conditioning and enviromental manipulations. Bebav. Res. and Tberapy 7, 165-171, 1969.

BACHRACH A. J., ERWIN, W. J., and MOHR, J. P.: "The control of eating behavior in an anorexic by operant conditioning techniques. Case Studies in Behavior Modifications. (Ed. by Ullman L. and Krasner L.) Holt, New York, 1965.

BiAnCO, J. B.: «Rapid treatment of two cases of anorexia nervosa». J. Bebav. Ther. and Exp. Psycbiat. S. 223-224, 1972.

Blinder, B. J., Freeman D. M., and Stunkard, A. J.: «Behavior therapy of anorexia nervosa: effectiveness of activity as reinforcer of weight gain). Am. J. Psycbiat. 126, 1093-1098, 1970.

BruCH, H.: «Perceptual and conceptual disturbances in anorexia nervosa». Psycbosom. Med. 24, 187, 1962.

BruCH, H.: «Disturbed communication in eating disorders». Am. J. Ortbopsycbiat, 33, 99-102, 1963.

BrUCH, H.: «Anorexia nervosa and its differential diagnosis», $J$. nerv. ment. Dis. 141, 55-556, 1966.

BruCH, H.: «Death in anorexia nervosa, Psychosom. Med. 33, 135-144, 1971.

BRUCH, H.: "Perils of behavior modification in treatment of anorexia nervosan. $J$. Am. med. Ass. 230, 1419-1422, 1974.

BrUCH, H.: "Anorexia nervosa: a review. Ross Timesaver: Feelings and their medical significance». NovDec, 1976, 1976.

HAILsten, E. A.: “Anorexia nervosa treated by desensitization». Bebav. Res and Tberapy, 3, 87-91, 1965.

LANG P. J.: «Behavior therapy with a case of anorexia nervosa. Case Studies in Behavior Modification» (Ed. by Ullman L. and Krasner L.). Holt. New York, 1965.

LeITENBERG, H., AgraS, W. S., and THOMPSON, L. E.: $\mu A$ sequential analysis of the effect of selective positive reinforcement in modifying anorexia nervosam. Bebav. Res. and Tberapy, 6, 211-218, 1968.

NEUMANN, M., and GAONI, B.: «Preferred food as the reinforcing agent in a case of anorexia nervosas. Bebav. Tber. and Exp. Psycbiat. 6, 331-333, 1975.

ShNuRER, A. T., Rubin, R. R., and RoY, A.: «Desensitization of anorexia nervosa seen as a weighn phobias. J. Bebav. Ther and Exp. Psysbiat. 4, 149-153, 1973.

StUMPhAUZER, J. S.: "Application of reinforcement contingencies with a 23-year-old anorexic patients. Psycb. Rep. 24, 109-110, 1969.

WOLPE, $J .:$ «Behavior therapy in anorexia nervosa and in general». J. Am. med. Ass. 233 (4), 1975. 